\title{
The Clinical Value of Implementing Early Rehabilitation in the Nursing of Patients with Cerebral Infarction
}

\author{
Weihua Xu* \\ Hubei No. 3 People's Hospital of Jianghan University, Wuhan City, Hubei Province, China \\ *Corresponding author: Weihua Xu,64784309@qq.com
}

\begin{abstract}
Objective: This research studied the clinical value of implementing early rehabilitation in the nursing of patients with cerebral infarction. Methods: Eighty-four patients with cerebral infarction in Hubei Third People's Hospital from January 2019 to January 2021 were selected and divided into a control group and a study group using the digital table method in which routine care and early rehabilitation care were given respectively and the effects were observed. Results: Prior to the nursing intervention, there was no significant difference in the National Institutes of Health Stroke Scale (NIHSS) and Barthel index between the two groups of patients, $\mathrm{P}>0.05$. After the nursing intervention, the NIHSS of the study group was lower than that of the control group whereas the Barthel index, Quality of Life Scale, and patient satisfaction in the study group were higher than the control group, $\mathrm{P}<0.05$. Conclusion: The implementation of early rehabilitation in the nursing of patients with cerebral infarction can effectively improve their neurological function and quality of life in addition to a higher satisfaction among them.
\end{abstract}

Keywords: Cerebral infarction; Early rehabilitation care; Clinical value

Publication date: July 2021; Online publication: July 31, 2021

\section{Introduction}

Cerebral infarction is also known as ischemic stroke in clinical practice. As a common cerebrovascular disease among middle-aged and elderly people, cerebral infarction is most often caused by abnormal blood supply mechanisms in the brain in which the brain is unable to obtain blood supply. This leads to the occurrence of ischemia and hypoxia, and eventually the brain tissues undergo avascular necrosis or softening. Hence, resulting in the disease which has high mortality and disability rate. ${ }^{[1]}$ In recent years, with the rapid advancement of medical technology, the treatment rate of patients with cerebral infarction has significantly increased but patients may still present with some sequelae after treatment such as hemiplegia or neurological damage which have great impact on their lives. Therefore, early nursing intervention is needed to improve these patients' quality of life. Based on this, the clinical value of early rehabilitation nursing was explored.

\section{Materials and methods}

\subsection{Background information}

This study included 84 patients with cerebral infarction in Hubei Third People's Hospital from January 2019 to January 2021. The inclusion criteria were patients that had undergone clinical examination to confirm the criteria for cerebral infarction and those that had voluntarily signed the informed consent form. The exclusion criteria were patients that had severe mental illness, cognitive dysfunction, dysfunction of 
vital organs, and non-cooperation. The patients were divided into a control group and a study group in which there were 42 cases in each group. The control group comprised of 22 male patients and 20 female patients, aging from 48 to 75 years old with an average age of $61.37 \pm 4.63$ years old while the study group comprised of 23 male patients and 19 female patients, aging from 49 to 74 years old with an average age of $61.45 \pm 4.71$ years old. Statistical software was used to analyze the patients' data in which no statistical difference was found $(\mathrm{P}>0.05)$.

\subsection{Method}

The control group was given routine nursing care which included post-treatment intervention, medication guidance and life guidance, etc. The study group was provided with early rehabilitation care:

(1) Positive care. In view that patients with cerebral infarction are prone for flexor reflexes, the nursing personnel provided targeted postural care. Nursing staffs were required to instruct patients to lie in bed and rest in a lateral position. Preventive care focusing on pressure sores and joint spasms were also included in which patients were regularly turned to minimize the duration in the same posture. In addition, massages were given at the compressed parts of the patients' body ${ }^{[2]}$.

(2) Psychological care. Patients that just experienced the disease would be unable to psychologically relieve themselves. Hence, the nursing staffs were required to actively communicate with the patients, accompany them, and appropriately provide information about successful nursing cases in order to build their confidence in nursing care and their compliance. At the same time, guidance in carrying out personalized daily living activities such as folding quilts, manicure, etc. was provided.

(3) Early functional exercise guidance. The nursing staffs were required to guide the patients to carry out various functional exercises. First, patients were instructed to perform passive movement of their joints and then gradually expand the scope. They would also carry out bilateral upper and lower extremity joint activities and turning movements. Secondly, swallowing function training was provided in addition to instructing the patients to perform daily masseter and facial muscle exercises 3 times a day, each lasting about 20 minutes. Finally, language function exercises for patients with aphasia were also provided in which a targeted training plan was developed such as dialogue training about 30 minutes a day. With the help of body language, patients would be able to understand the meaning and gradually learn to pronounce. At the same time, patients' family members were encouraged to talk to the patient so that the patients' language function could recover quickly.

(4) Discharge guidance. After the patient had been discharged, the nursing staffs followed up once every six months or once a year via telephone or on-site guidance to follow-up with their recovery. At the same time, post-hospital related precautions were provided and the patients were also encouraged to develop good habits to promote rapid recovery of their body.

\subsection{Evaluation}

The improvement of the patients' neurological function and living ability before and after nursing were observed. The neurological function was evaluated by the National Institutes of Health Stroke Scale (NIHSS) in which the higher the score, the more severe the neurological impairment of the patient. Barthel index was also used to evaluate the daily activity in which the higher it was, the higher the patient's ability to live. The Quality of Life Scale was used to evaluate the improvement of the patient's quality of life after care mainly in terms of strength, mobility, hand function, emotion, communication, memory and thinking, social participation, and others. The higher the score, the higher the quality of life. ${ }^{[4]}$

Patient satisfaction was evaluated by a self-made questionnaire provided to the patients by the hospital. The questionnaire had a total of 100 points and had three levels. If the evaluation score exceeded 90 points, 
it belonged to the first level indicating very satisfied. 70-90 points which belonged to the second level indicated basically satisfied, and scores below 70 points belonged to the third level indicating dissatisfaction. The total satisfaction $=($ very satisfied + basically satisfied $) /$ total number of cases $\times 100 \% .{ }^{[5]}$

\subsection{Statistical analysis}

The data were processed and analyzed by Statistical Package for the Social Sciences (SPSS) version 23.0 software, using t-test and chi-square $\left(\mathrm{X}^{2}\right)$ for testing, and expressed by $(\bar{x} \pm \mathrm{s})$ and $(\%) . \mathrm{P}<0.05$ indicated differences in the statistical data.

\section{Results}

\subsection{Improvement of neurological function and living ability}

According to the scoring statistics shown in Table 1., there was no significant difference in the scores of the two groups before nursing. After nursing, the neurological function and living ability of the patients effectively improved. The improvement effect was higher in the study group, $\mathrm{P}<0.05$.

Table 1. Statistics of NIHSS score and Barthel index $(\bar{x} \pm s)$

\begin{tabular}{cccccc}
\hline \multirow{2}{*}{ Group } & \multirow{n}{*}{ NIHSS } & \multicolumn{2}{c}{ Barthel } \\
\cline { 3 - 6 } & & Before care & After care & Before care & After care \\
\hline Control group & 42 & $24.53 \pm 3.35$ & $17.24 \pm 2.71$ & $50.28 \pm 5.13$ & $69.58 \pm 5.36$ \\
Study group & 42 & $24.46 \pm 3.31$ & $11.17 \pm 2.92$ & $50.25 \pm 5.24$ & $78.75 \pm 6.99$ \\
t & & 0.0963 & 9.8746 & 0.0265 & 6.7467 \\
$\mathrm{P}$ & & 0.9235 & 0.0000 & 0.9789 & 0.0000 \\
\hline
\end{tabular}

\subsection{Quality of life}

As shown in Table 2., the quality of life scores in the study group were higher than those in the control group, $\mathrm{P}<0.05$

Table 2. Statistics of the quality of life scores $(\bar{x} \pm s)$

\begin{tabular}{ccccc}
\hline Quality of life & Control group $(\mathbf{n}=\mathbf{4 2})$ & Study group $(\mathbf{n}=\mathbf{4 2})$ & $\mathbf{t}$ & $\mathbf{P}$ \\
\hline Power & $85.34 \pm 3.61$ & $94.17 \pm 2.87$ & 12.4083 & 0.0000 \\
Hand function & $84.64 \pm 4.11$ & $92.25 \pm 2.61$ & 10.1297 & 0.0000 \\
Action ability & $87.52 \pm 3.59$ & $93.07 \pm 2.54$ & 8.1789 & 0.0000 \\
Memory and thinking & $86.64 \pm 3.67$ & $92.58 \pm 2.05$ & 9.1575 & 0.0000 \\
Communication & $87.79 \pm 4.41$ & $93.51 \pm 2.52$ & 7.2983 & 0.0000 \\
Emotion & $89.12 \pm 4.25$ & $94.55 \pm 3.51$ & 6.3843 & 0.0000 \\
Social participation & $88.02 \pm 4.21$ & $95.87 \pm 2.33$ & 10.5728 & 0.0000 \\
\hline
\end{tabular}

\subsection{Patient satisfaction}

The questionnaire survey showed that the patient satisfaction of the study group was higher than that of the control group, $\mathrm{P}<0.05$. 
Table 3. Statistics of patient satisfaction $(\%)$

\begin{tabular}{cccccc}
\hline Group & n & Very satisfied & Basically satisfied & Dissatisfied & Total satisfaction \\
\hline Control group & 42 & 14 & 19 & 9 & 75.57 \\
Study group & 42 & 16 & 24 & 2 & 95.23 \\
$\mathrm{X}^{2}$ & & & & 5.1258 \\
$\mathrm{P}$ & & & & & 0.0235 \\
\hline
\end{tabular}

\section{Discussion}

Cerebral infarction is a relatively common cerebrovascular disease. Although the current clinical treatment rate of patients has significantly improved, it usually takes a long time for patients to recover after treatment. During the recovery period, there may be some dysfunctions, nerve defects, etc. Therefore, in order to promote the rapid recovery of patients and avoid the occurrence of undesirable situations, it is extremely important to provide early nursing interventions ${ }^{[6]}$.

According to related studies, the application of early rehabilitation in nursing has an extremely important role in the intervention of cerebral infarction. It is a more comprehensive and systematic nursing method in the current clinical practice. It intervenes the patients' situation and psychology to improve their psychological status and reduces the risk of complications. In addition to that, based on the specific recovery of the patients, a personalized early rehabilitation exercise plan can be formulated in guiding the patients to conduct various functional exercises, hence improving their neurological and physical function. Finally, discharge guidance can be provided to help establish patients' management of self-awareness and to promote their rapid recovery ${ }^{[7-8]}$. From these results, the improvement of the patients in the study group in terms of their neurological function and activities of daily living were obviously higher than that in the control group. Their quality of life and satisfaction were also higher than those in the control group. The contrast between the groups was significantly different.

\section{Disclosure statement}

The author declares no conflict of interest.

\section{References}

[1] Bian G, 2019, Clinical nursing effect evaluation and application value analysis of implementing early rehabilitation nursing mode in cerebral infarction nursing. Health for All, 507(22): 163.

[2] Yang JJ, 2019, The Application of Early Rehabilitation Nursing Model in Clinical Nursing of Cerebral Infarction Patients was Analyzed. Systems Medicine.

[3] Qiu X, Zhang Y, Hu X, 2020, Application effect analysis of early rehabilitation nursing model in clinical nursing of patients with cerebral infarction. Electronic Journal of Practical Clinical Nursing, 5(16): 96, 106.

[4] Zhang YM, Xin ZF, Pei HF, et al., 2019, Effect of Path-type Early Rehabilitation Nursing on the Recovery of Upper Limb Function in Patients with Cerebral Infarction. Heilongjiang Medical Journal.

[5] Chen Y, 2020, Analyze the application effect and life impact of early rehabilitation nursing model in elderly patients with cerebral infarction. The World's Latest Medical Information, 20(71): 286-7.

[6] Jing GU, 2019, Effect of Acupuncture Combined with Early Rehabilitation Nursing on Patients with Acute Cerebral Infarction. Biped and Health.

[7] Cong L, 2019, Evaluation of the clinical effect of early rehabilitation nursing mode and the impact of nursing satisfaction in patients with cerebral infarction. Electronic Journal of Clinical Medicine, 6(12): 134.

[8] Huang L, 2020, Study on the clinical value of early rehabilitation nursing for improving the nursing compliance and motor function of patients with cerebral infarction. Diet Health Care, 007(011): 206-7. 